\title{
Ácaros (Arachnida, Acari) da seringueira (Hevea brasiliensis Muell. Arg.) no Estado do Mato Grosso, Brasil
}

\author{
Noeli Juarez Ferla ${ }^{1}$ \\ Gilberto José de Moraes ${ }^{2}$
}

\begin{abstract}
Mites (Arachnida, Acari) on rubber tree (Hevea brasiliensis Muell. Arg.) in the State of Mato Grosso, Brazil. Mites started to be considered economically important on rubber tree since the early 1990 's, when they were considered responsible for damages caused to this crop and by the consequent yield reduction. The aim of this work was to determine the mites present on this crop in the State of Mato Grosso and to elaborate a key for the separation of the species found. This study was conducted in fields of Plantações Edouard Michelin Ltda., in Itiquira, and Triângulo Agro-Industrial S/A, in Pontes e Lacerda. Samplings were conducted monthly in six different clones: PB 260, PR 255, IAN 713, IAN 873, FX 3864 and RRIM 600. Clones PB 260 and IAN 873 were sampled between August 1998 and July 2000; other clones were sampled between August 1999 and July 2000. In the season 1998/1999 samples consisted of 15 leaves of each of 10 plants taken randomly and in the season 1999/2000, 5 leaves of the median stratum of each of 15 plants taken randomly were sampled. A total of 4270 mites were found, belonging to 11 families. Nearly $77,2 \%$ of the mites collected belonged to the families Eriophyidae, Tarsonemidae, Tenuipalpidae and Tetranychidae, composed predominantly by phytophagous species. Phytoseiidae was the most diverse family, with 15 species, followed by the families Tetranychidae and Eriophydae, with five species, and Tydeidae, with four species

KEY WORDS. Acari, mites, diversity of mite, Hevea brasiliensis, rubber tree, Mato Grosso
\end{abstract}

A seringueira, Hevea brasiliensis Muell. Arg. (Euphorbiaceae), é comumente atacada por diversas espécies de insetos e ácaros nas áreas onde é cultivada, sendo que algumas são consideradas pragas de suma importância (SILVA 1972; EMBRATER 1983). Os ácaros passaram a ser considerados economicamente importantes a partir da década de 1990, quando foram responsabilizados por danos causados a esta cultura.

Os ácaros fitófagos, comumente encontrados, pertencem às famílias Eriophyidae, Tarsonemidae, Tenuipalpidae e Tetranychidae, enquanto que, entre os predadores, os ácaros mais comuns pertencem às famílias Cunaxidae, Phytoseiidae e Stigmaeidae (Silva 1972; FleChtMAnN 1989; FleChtManN \& ARLeu 1984; FAZOLIN \& PEREIRA 1989; FERES 1992, 1998, 2000).

1) Museu de Ciências Naturais, Centro Universitário UNIVATES. 95900-000 Lajeado, Rio Grande do Sul, Brasil. E-mail: njferla@fates.tche.br

2) Departamento de Entomologia, Fitopatologia e Zoologia Agrícola, Escola Superior de Agricultura Luiz de Queiroz. 13418-900 Piracicaba, São Paulo, Brasil. Bolsista do CNPq. E-mail: gjmoraes@carpa.ciagri.usp.br 
Poucos estudos foram realizados para o reconhecimento de ácaros na cultura da seringueira até a década de 1990. Até a década de 1940 apenas Tenuipalpus heveae Baker havia sido descrito (BAKER 1945). Desde então, outros artigos sobre as espécies presentes nesta cultura foram publicados, destacando-se os trabalhos de SILVA (1972), Flechtmann \& ARleu (1984), Fazolin \& Pereira (1989) e FleChtManN (1989).

Devido ao dano e à perda de produtividade dos seringais, atribuídos às altas infestações de ácaros, principalmente por Calacarus heveae Feres, 1992, vários estudos foram iniciados, a partir da década de 1990 , com o intuito de reconhecer as principais espécies e os danos causados por elas nos seringais, naqueles estados em que esta cultura tinha sido introduzida. Três espécies de eriofídeos foram descritas: C. heveae, Phyllocoptruta seringueirae Feres, 1998 e Schevtchenkella petiolula Feres, 1998). FERES (2000) reportou 28 espécies de ácaros pertencentes a 24 gêneros de 11 famílias presentes na cultura da seringueira nos Estados do Mato Grosso, Mato Grosso do Sul, Minas Gerais e São Paulo.

O objetivo deste trabalho foi ampliar os conhecimentos sobre a diversidade da fauna acarológica presente na cultura da seringueira no Estado do Mato Grosso e elaborar uma chave dicotômica para a separação das espécies encontradas.

\section{MATERIAL E MÉTODOS}

Este estudo foi conduzido em campos de seringueira das Plantações Edouard Michelin Ltda., no município de Itiquira, e da Triângulo Agro-industrial S.A., no município de Pontes e Lacerda, Mato Grosso.

As coletas foram feitas mensalmente em seis clones suscetíveis aos ácaros. Em Itiquira, foram escolhidos os clones PB 260 (4,5 ha e 12 anos) e PR 255 (6,4 ha e 15 anos), e, em Pontes e Lacerda, os clones IAN 713 (2,6 ha e 15 anos), IAN 873 (17,1 ha e 16 anos), FX 3864 ( 6,5 ha e 14 anos) e RRIM 600 (3,2 ha e 15 anos). Para os clones PB 260 e IAN 873, as coletas foram realizadas de agosto de 1998 a julho de 2000; para os clones PR 255, IAN 713, FX 3864 e RRIM 600, as coletas foram realizadas de agosto de 1999 a julho de 2000.

Na safra 1998/1999, a amostragem consistiu de três folhas dos estratos basal, mediano e apical, totalizando 15 folhas, coletadas de cada uma das 10 plantas escolhidas ao acaso em cada campo, localizadas pelo menos a $20 \mathrm{~m}$ da borda. Os campos apresentavam aproximadamente 500 plantas com altura em torno de 20 metros. Na segunda safra, entre 1999/2000, cinco folhas de cada uma das 15 plantas escolhidas foram amostradas do estrato mediano nos seis clones IAN 713, IAN 873, FX 3864, PB 260, PR 255 e RRIM 600.

Para a identificação das espécies acarinas foram tomadas amostras de $25 \%$ das folhas. Dez ácaros de cada família foram coletados, com a utilização de pincel de ponta fina. Para exame ao microscópio, os eriofídeos foram montados em meio modificado de Berlese (AMRINE \& MANSON 1996) e os demais ácaros coletados foram montados usando meio de Hoyer (FLECHTMANN 1975). As lâminas montadas foram mantidas em estufa a $50-60^{\circ} \mathrm{C}$ por cerca de 10 dias para a fixação, distensão e clarificação dos espécimes e secagem do meio. Posteriormente, foi feita a lutagem das bordas das lamínulas. 
Para os fitoseídeos, utilizou-se o sistema de classificação genérico proposto por Chant \& Yoshida-Shaul (1983), Chant \& Yoshida-Shaul (1986a, b), CHANT \& MCMURTRY (1994) e MORAES et al. (1986).

Espécimes representantes de cada uma das espécies encontradas foram depositados na Coleção de Referência de Ácaros, Setor de Zoologia, Departamento de Entomologia, Fitopatologia e Zoologia Agrícola da Escola Superior de Agricultura, Universidade de São Paulo (ESALQ-USP), Piracicaba, São Paulo.

\section{RESULTADOS}

Foi encontrado um total de 4.270 ácaros pertencentes a 11 famílias nas plantas analisadas. A maioria dos indivíduos coletados, cerca de 3.300 ácaros, pertencia às famílias Eriophyidae, Tarsonemidae, Tenuipalpidae e Tetranychidae, compostas por espécies predominantemente fitófagas. Dos ácaros restantes, 838 indivíduos pertenciam às famílias Cheyletidae, Cunaxidae, Phytoseiidae e Stigmaeidae, compostas por espécies predominantemente predadoras e 122 às famílias Tydeidae e Acaridae, de hábitos polífagos.

Os ácaros encontrados pertencem a 41 espécies distintas. A família Phytoseiidae apresentou maior diversidade, com 15 espécies, seguida pelas famílias Tetranychidae e Eriophyidae, com cinco espécies cada, e a família Tydeidae, com quatro espécies.

São apresentados, a seguir, os grupos de ácaros encontrados, o mês e o ano da coleta e o número total de machos e fêmeas em cada clone.

\section{Subordem Gamasida Phytoseiidae Berlese, 1913}

\section{Amblyseius acalyphus Denmark \& Muma, 1973}

Amblyseius acalyphus Denmark \& Muma, 1973: 243; 1989: 75; Feres \& Moraes, 1998: 125.

Espécimes examinados. Itiquira: PB 260 V-99, IV-00, V-00, VI-00 (22 fêmeas e 4 machos); PR 255 V-00 (1 fêmea); Pontes e Lacerda: IAN 713 XI-99 (4 fêmeas e 1 macho); FX 3864 X-99 (2 fêmeas).

Registros prévios no Brasil. São Paulo (Feres \& MORAes 1998).

Observações. As medidas conferem com as medidas relatadas por DENMARK \& Muma (1989), exceto pelo comprimento menor de Z5 e macroseta do genu IV (220 $\mu \mathrm{m}$ e $86 \mu \mathrm{m}$ para o holótipo e $175 \mu \mathrm{m}$ e $73 \mu \mathrm{m}$, respectivamente, para os espécimes examinados). É a primeira citação desta espécie nesta cultura.

\section{Amblyseius neochiapensis Lofego, Moraes \& McMurtry, 2000}

Amblyseius neochiapensis Lofego, Moraes \& McMurtry, 2000: 462.

Espécimes examinados. Itiquira: PB 260 V-00 (1 fêmea); Pontes e Lacerda: IAN 873 XII-98, IV-99, V-99, VIII-99, IX-99 (11 fêmeas e 3 machos); IAN 713 VIII-99, IX-99, X-99, XI-99 (7 fêmeas e 2 machos); FX 3864 VIII-99, IX-99, X-99, XI-99, XII-99, I-00, VI-00 (10 fêmeas e 4 machos); RRIM 600 VIII-99, IX-99 (3 fêmeas).

Registros prévios no Brasil. São Paulo (LOFEGo et al. 2000).

Observação. É a primeira citação desta espécie nesta cultura. 


\section{Euseius alatus DeLeon, 1966}

Euseius alatus DeLeon, 1966: 87; Denmark \& Muma, 1973: 262; Moraes \& McMurtry, 1983: 137; Feres \& Moraes, 1998: 127.

Euseius paraguayensis Denmark \& Muma, 1970: 224 (sinonímia de acordo com Moraes \& McMurtry, 1983: 137).

Espécimes examinados. Itiquira: PR 255 I-00 (1 fêmea); Pontes e Lacerda: IAN 873 IX-98, XI-98, VII-99, IX-99, X-99, XI-99, XII-99 (32 fêmeas e 6 machos); IAN 713 IX-99, X-99, XI-99, XII-99 (8 fêmeas e 1 macho); FX 3864 IX-99, X-99, XI-99, XII-99 (7 fêmeas); RRIM 600 IX-99, X-99, XII-99 (5 fêmeas e 1 macho).

Registros prévios no Brasil. Bahia, Ceará, Maranhão, Mato Grosso do Sul, Minas Gerais, Paraíba, Pernambuco e Rio Grande do Sul (MoraES et al. 1986; Feres \& MORAES 1998; FERla \& MORAES 1998).

Observações. REIS et al. (2000) sugerem que esta espécie seja predadora de Phyllocoptruta oleivora (Ashmead) na cultura de citros. É a primeira citação de $E$. alatus na cultura da seringueira.

\section{Euseius citrifolius Denmark \& Muma, 1970}

Euseius citrifolius Denmark \& Muma, 1970: 222; Moraes \& McMurtry, 1983: 128; Feres \& Moraes, 1998: 125; Feres, 2000: 161.

Espécimes examinados. Itiquira: PB 260 IX-98, II-99, III-99, V-99, XI-99 (19 fêmeas e 3 machos); PR 255 XI-99, I-00 (4 fêmeas).

Registros prévios no Brasil. Bahia, Ceará, Maranhão, Mato Grosso do Sul, Minas Gerais, Paraíba, Pernambuco, Piauí, Rio Grande do Sul e São Paulo (Moraes et al. 1986; Feres \& Moraes 1998; FERES 2000; Ferla \& MoraES 1998).

Observações. Sua presença na cultura da seringueira foi relatada por FERES (2000), nos Estados de Mato Grosso e São Paulo.

\section{Euseius concordis (Chant, 1959)}

Typhlodromus (Amblyseius) concordis Chant, 1959: 69.

Amblyseius (Iphiseius) concordis; Muma, 1961: 288.

Amblyseius concordis; Chant \& Baker, 1965: 22; Moraes \& McMurtry, 1983: 138.

Euseius concordis; Denmark \& Muma, 1973: 264; Moraes \& Oliveira, 1982: 317; Moraes \& McMurtry, 1983: 138; Feres \& Moraes, 1998: 127

Euseius flechtmanni; Denmark \& Muma, 1970: 223; Denmark \& Muma, 1973: 261 (sinonímia de acordo com Moraes et al., 1982: 18).

Espécimes examinados. Itiquira: PB 260 IX-98, XII-98, I-99, III-99, V-99, VIII-99, I-00, IV-00, V-00, VI-00, VII-00 (32 fêmeas e 8 machos); PR 255 VIII-99, VII-00 (14 fêmeas e 3 machos); Pontes e Lacerda: IAN 873 IX-98, VII-99, VIII-99, IX-99, X-99, I-00 (46 fêmeas e 5 machos); IAN 713 VIII-99, IX-99, X-99, XI-99, XII-99, I-00, V-00 (84 fêmeas e 8 machos); FX 3864 VIII-99, IX-99, X-99, I-00, V-00 (20 fêmeas e 4 machos); RRIM 600 VIII-99, IX-99, X-99, XI-99, XII-99, I-00, III-00, V-00 (67 fêmeas e 12 machos).

Registros prévios no Brasil. Bahia, Ceará, Maranhão, Mato Grosso do Sul, Paraíba, Pernambuco, Piauí, Rio Grande do Sul e São Paulo (MORAes et al. 1986; FERES \& MoraES 1998; FERLA \& MORAES 1998). 
Observações. Uma das espécies mais comumente encontradas em plantas no Brasil e em outros países da América do Sul (MORAES 1991). É uma das espécies mais frequientes em citros, sendo estudada para o manejo integrado de pragas nesta cultura (GRAVENA 1994). Foi relatada por FERES (2000) na cultura da seringueira no Estado de Mato Grosso. No presente estudo, foi a espécie de Phytoseiidae mais freqüentemente encontrada.

\section{Galendromimus alveolaris (DeLeon, 1957)}

Typhlodromus alveolaris DeLeon, 1957: 141.

Typhlodromus (Typhlodromus) alveolaris; Chant, 1959: 52.

Cydnodromella alveolaris; Chant \& Yoshida-Shaul, 1986b: 2821; Moraes \& Mesa, 1988: 80.

Galendromimus alveolaris; DeLeon, 1962b: 175; DeLeon, 1967:13; Muma, 1961: 298; Muma et al., 1970: 58.

Galendromimus (Galendromimus) alveolaris; Moraes et al., 2000: 255.

Espécimes examinados. Pontes e Lacerda: RRIM 600 VII-00 (2 fêmeas).

Observação. Esta é a primeira constatação desta espécie na cultura da seringueira.

\section{Galendromus annectens DeLeon, 1958}

Typhlodromus annectens DeLeon, 1958: 75; Moraes \& McMurtry, 1983: 142; Chant \& Yoshida-Shaul, 1984: 1868; Moraes \& Mesa, 1988: 82; Moraes et al. 1991: 134; Feres \& Moraes, 1998: 129.

Galendromus annectens; Muma, 1961: 298; Muma et al. 1970: 135; Denmark \& Muma, 1973: 274; Farias et al. 1981: 316; Moraes et al. 1982: 21.

Espécimes examinados. Itiquira: PB 260 X-99 (1 fêmea); PR 255 X-99 (1 fêmea); Pontes e Lacerda: IAN 713 XI-99, XII-99, I-00 (6 fêmeas e 1 macho); RRIM 600 IX-99 (1 fêmea).

Registros prévios no Brasil. Pernambuco, São Paulo e Rio Grande do Sul (Moraes et al. 1986; Feres \& Moraes 1998; Ferla \& Moraes 1998, Feres 2000).

Observações. Espécie já foi relatada nesta cultura por FERES (2000) no Estado de Mato Grosso.

\section{Galendromus sp.}

Espécimes examinados. Pontes e Lacerda: IAN 873 VII-99 (10 fêmeas).

\section{Neoseiulus anonymus (Chant \& Baker, 1965)}

Amblyseius anonymus Chant \& Baker, 1965: 21; Schicha \& Elshafie, 1980: 32; McMurtry, 1983: 254. Neoseiulus anonymus; Denmark \& Muma, 1973: 27; Moraes \& Mesa, 1988: 76; Moraes et al. 1991: 126; Kreiter \& Moraes, 1997: 378; Moraes et al. 2000: 245.

Espécimes examinados. Itiquira: PB 260 X-98, XI-98, XII-98, XII-99, I-00 (37 fêmeas e 3 machos); PR 255 XII-99 (13 fêmeas e 2 machos). Pontes e Lacerda: IAN 873 X-98, XI-98, XII-98, X-99, XI-99, XII-99 (17 fêmeas e 3 machos); IAN 713 XI-99, XII-99 (5 fêmeas); FX 3864 XII-99 (1 fêmea); RRIM 600 IX-99, X-99, XII-99, II-00 (5 fêmeas e 1 macho).

Registros prévios no Brasil. Paraná e São Paulo (MORAES et al. 1986). 
Observações. É uma das espécies comumente associadas à Mononychellus tanajoa (Bondar) no Nordeste do Brasil (MORAES et al. 1988). No presente estudo, foi a segunda espécie mais freqüentemente encontrada.

\section{Neoseiulus tunus (DeLeon, 1967)}

Typhlodromips tunus DeLeon, 1967: 29; Denmark \& Muma, 1973: 253.

Amblyseius tunus; Feres \& Moraes, 1998: 126.

Espécimes examinados. Pontes e Lacerda: IAN 713 X-99 (1 fêmea).

Registros prévios no Brasil. Rio Grande do Sul, Santa Catarina e São Paulo (MORAES et al. 1986; Feres \& MORAES 1998; Ferla \& MORAES 1998).

Observações. Esta espécie foi comumente encontrada em pomares não tratados de macieira, no Estado do Rio Grande do Sul (FERLA \& MORAES 1998). É a primeira constatação desta espécie em seringueira.

\section{Typhlodromalus aff. horatii}

Espécimes examinados. Pontes e Lacerda: FX 3864 X-99 (1 fêmea).

\section{Typhlodromalus feresi Lofego, Moraes \& McMurtry, 2000}

Typhlodromalus feresi Lofego, Moraes \& McMurtry, 2000: 466.

Espécimes examinados. Pontes e Lacerda: IAN 873 X-99 (1 fêmea).

Registros prévios no Brasil. São Paulo (LOFEGO et al. 2000).

Observações. O espécime encontrado apresentou setas j3, z4, s4, J2, Z1, Z4, Z5, S2, S4, r3 menores que o holótipo, enquanto que as macrosetas do genu I, II, III e do tarso IV são maiores que as macrosetas do holótipo. É a primeira citação desta espécie nesta cultura.

\section{Typhlodromips amilus DeLeon, 1967}

Typhlodromips amilus DeLeon, 1967: 28.

Espécimes examinados. Pontes e Lacerda: IAN 873 VII-99, VIII-99 (5 fêmeas); IAN 713 XII-99, I-00, IV-00, VI-00 (5 fêmeas e 1 macho); FX 3864 VIII-99, X-99, XI-99, XII-99, I-00, VI-00 (11 fêmea e 3 machos); RRIM 600 VII-99 (1 fêmea).

Observações. Macrosetas das pernas maiores que as macrosetas de T. amilus e o cérvix da espermateca é menor que o de $T$. amilus. É a primeira citação desta espécie no Brasil.

\section{Typhlodromips aff. sinensis}

Espécimes examinados. Pontes e Lacerda: IAN 873 IV-99, V-99 (7 fêmeas e 2 machos).

\section{Typhlodromus (Anthoseius) transvaalensis (Nesbitt, 1951)}

Kampimodromus transvaalensis Nesbitt, 1951: 55.

Typhlodromus transvaalensis, Chant, 1955: 498.

Espécimes examinados. Pontes e Lacerda: IAN 873 V-99 (1 fêmea). 
Registros prévios no Brasil. São Paulo e Rio Grande do Sul (MORAES et al. 1986; FERLA \& MORAES 1998).

Observação. É a primeira constatação desta espécie na cultura da seringueira.

\section{Subordem Acaridida \\ Acaridae Ewing \& Nesbitt, 1954 \\ Tyrophagus sp.}

Espécimes examinados. Itiquira: PB 260 V-99, V-00 (3 fêmeas); PR 255 V-00 (1 fêmea).

\section{Neotropacarus sp.}

Espécimes examinados. Itiquira: PB 260 V-00, VI-00, VII-00 (10 fêmeas).

Wintershmidtiidae Oudemans, 1923

\section{Oulenzia sp.}

Espécimes examinados. Itiquira: PB 260 II-99, V-99, I-00 (4 fêmeas); PR 255 IV-00, V-00, VI-00 (3 fêmeas).

\section{Subordem Actinedida Cheyletidae Leach, 1815}

\section{Hemicheyletia sp.}

Espécimes examinados. Itiquira: PB 260 III-99 (1 fêmea); Pontes e Lacerda: IAN 873 II-99 (1 fêmea).

Observações. São ácaros predadores que se utilizam de emboscada para atacar suas presas (Perrin \& MCMurTRy 1996). Segundo Chiavegato (1980), Hemicheyletia welsi (Baker) tem sido coletada com facilidade em folhas e frutos de citros atacados pelo ácaro da falsa ferrugem (Phyllocoptruta oleivora (Ashmead)). FERES (2000) relatou a presença de $H$. welsi em seringais do Estado de São Paulo.

\section{Cunaxidae Thor, 1902}

\section{Pseudobonzia sp.}

Espécimes examinados. Itiquira: PB 260 II-00 (1 fêmea); PR 255 II-00 (1 fêmea); Pontes e Lacerda: IAN 873 X-98, XII-98, II-99, III-99, IV-99, V-99, VII-99, VIII-99, I-00 (45 fêmeas e 15 machos); IAN 713 VIII-99, I-00, VI-00 (4 fêmeas); FX 3864 VIII-99, XI-99, XII-99, I-00, II-00, III-00, IV-00, V-00, VI-00 (52 fêmeas e 8 machos); RRIM 600 VIII-99, XII-99, V-00 (16 fêmeas e 4 machos).

Observações. Ácaros da família Cunaxidae são predadores de outros ácaros e de pequenos artrópodes (SMILey 1992; Perrin \& MCMurTRY 1996). Pseudobonzia sp. foi relatado por FERES (2000) em seringais do Estado do Mato Grosso. 


\section{Eriophyidae Nalepa, 1898}

Aff. Acaphyllisa sp.

Espécimes examinados. Itiquira: PB 260 XII-99 (1 fêmea).

Observação. Este espécime, por aparentar empódio dividido, pode pertencer a um novo gênero, próximo de Acaphyllisa Keifer (Reinaldo Feres, comunicação pessoal).

\section{Aff. Chakrabartiella sp.}

Espécimes examinados. Pontes e Lacerda: FX 3864 V-00, VI-00 (4 fêmeas).

Observações. Os espécimes pertencem possivelmente a um novo gênero, próximo de Chakrabartiella Amrine \& Stasny (Reinaldo Feres, comunicação pessoal).

\section{Calacarus heveae Feres, 1992}

Calacarus heveae Feres, 1992: 61; Feres, 2000: 167.

Espécimes examinados. Itiquira: PB 260 XII-98, I-99, II-99, III-99, V-99, VIII-99, XI-99, XII-99, I-00, II-00, V-00 (260 fêmeas e 15 machos); PR 255 VIII-99, XI-99, XII-99, I-00, II-00, V-00, VI-00 (26 fêmeas e 4 machos); Pontes e Lacerda: IAN 873 X-98, XI-98, XII-98, II-99, III-99, XII-99, II-00, VII-00 (103 fêmeas e 8 machos); IAN 713 III-00, IV-00, V-00 (25 fêmeas e 4 machos); FX 3864 IV-00, V-00 (9 fêmeas); RRIM 600 I-00, II-00, III-00, V-00 (78 fêmeas e 9 machos).

Registros prévios no Brasil. Mato Grosso, Mato Grosso do Sul, Minas Gerais e São Paulo (FERES 2000).

Observações. Espécie descrita de material coletado da região noroeste do Estado de São Paulo. Prefere a face adaxial dos folíolos e causa perda do brilho, amarelecimento e bronzeamento das folhas de seringueira e sua conseqüente queda prematura (FERES 2000).

\section{Phyllocoptruta seringueirae Feres, 1998}

Phyllocoptruta seringueirae Feres, 1998: 71; Feres, 2000: 168.

Espécimes examinados. Itiquira: PB 260 VIII-99, XI-99, I-00, II-00, VII-00 (10 fêmeas e 1 macho)); PR 255 VIII-99, XI-99, II-00, IV-00, V-00, VII-00 (9 fêmeas e 2 machos); Pontes e Lacerda: IAN 873 XI-98, XII-98, III-99, IV-99, V-99, VIII-99, IX-99, XI-99, XII-99, I-00, II-00, III-00, IV-00, V-00, VI-00, VII-00 (280 fêmeas e 25 machos); IAN 713 VIII-99, IX-99, X-99, XI-99, XII-99, I-00, II-00, III-00, IV-00, V-00, VI-00, VII-00 (192 fêmease 18 machos); FX 3864 VIII-99, IX-99, X-99, XII-99, I-00, II-00, IV-00, V-00 (285 fêmeas e 15 machos); RRIM 600 VIII-99, IX-99, X-99, XI-99, XII-99, I-00, II-00, III-00, IV-00, V-00 (180 fêmeas e 17 machos).

Registros prévios no Brasil. Mato Grosso, Mato Grosso do Sul e São Paulo (FERES 2000).

Observações. Espécie de cor alaranjada, que ocorre na face adaxial e abaxial do folíolo (FERES 2000). 


\section{Shevtchenkella petiolula Feres, 1998}

Shevtchenkella petiolula Feres, 1998: 69; Feres, 2000: 168.

Espécimes examinados. Itiquira: PB 260 X-99, XI-99 (4 fêmeas); PR 255 X-99 (2 fêmeas); Pontes e Lacerda: IAN 873 X-98, XI-98, XII-98, V-99, XI-99, V-00, VII-00 (20 fêmeas e 3 machos); IAN 713 XII-99, II-00 (3 fêmeas); FX 3864 IX-99, X-99, XII-99, I-00, II-00, IV-00, V-00 (20 fêmeas e 1 macho); RRIM 600 IX-99, X-99, XII-99 (11 fêmea e 2 machos).

Registros prévios no Brasil. Mato Grosso, Mato Grosso do Sul e São Paulo (FERES 2000).

Observações. Espécie de cor alaranjada, encontrada preponderantemente em pecíolos e peciólulos das folhas de seringueira (FERES 2000).

\section{Tarsonemidae Kramer, 1877}

\section{Polyphagotarsonemus latus (Banks, 1904)}

Tarsonemus latus Banks, 1904: 1553.

Hemitarsonemus latus; Ewing, 1939: 54.

Neotarsonemus latus; Smiley, 1967: 137.

Espécimes examinados. Itiquira: PB 260 X-99 (1 fêmea); Pontes e Lacerda: IAN 873 X-99 (2 fêmeas); FX 3864 IX-99 (2 fêmeas); RRIM 600 IX-99 (1 macho).

Observações. Conhecido como ácaro branco ou tropical, praga de diversas culturas. A primeira citação desta espécie na cultura da seringueira foi feita por FLECHTMANN \& ARLEU (1984). Segundo os mesmos autores, o ataque desta espécie restringe-se aos brotos das plantas, causando deformação e bronzeamento das folhas.

\section{Tarsonemus sp.}

Espécimes examinados. Itiquira: PB 260 I-99, XI-99, I-00, II-00 (17 fêmeas e 1 macho); PR 255 XI-99, I-00, II-00 (12 fêmeas); Pontes e Lacerda: IAN 873 IV-99, V-99 (1 macho); RRIM 600 II-00 (1 fêmea).

Observações. Ácaros deste gênero são primariamente micófagos (LINDQUIST 1986).

\section{Tenuipalpidae Berlese, 1913}

\section{Brevipalpus phoenicis (Geijskes, 1939)}

Tenuipalpus phoenicis Geijskes, 1939: 23.

Brevipalpus phoenicis; Sayed, 1946: 99; Pritchard \& Baker, 1958: 223; DeLeon, 1961: 48; Gonzales,

1975: 82; Baker et al. 1975: 18; Meyer, 1979: 87; Baker \& Tuttle, 1987: 98; Feres, 2000: 164.

Espécimes examinados. Itiquira: PB 260 II-00 (2 fêmeas); Pontes e Lacerda: IAN 873 II-99 (1 fêmea), FX 3864 VIII-99 (1 fêmea).

Registros prévios no Brasil. Alagoas, Bahia, Ceará, Minas Gerais, Paraná, Pernambuco, Rio de Janeiro e São Paulo (FleCHTMAnN 1976). 
Observações. Espécie de ampla distribuição geográfica, ocorrendo sobre grande número de espécies hospedeiras (FERES 2000). É conhecido como "ácaroda-leprose-dos-citros", pois é vetor da virose que causa essa patogenia nos citros (CHIAVEGATO 1980). No Brasil, foi registrado pela primeira vez em seringueira por FLECHTMANN \& ARLEU (1984).

\section{Tenuipalpus heveae Baker, 1945}

Tenuipalpus heveae Baker, 1945: 36; Baker \& Pritchard, 1953: 320; Feres, 2000: 165.

Espécimes examinados. Itiquira: PB 260 IX-98, XII-98, I-99, II-99, III-99, IV-99, V-99, X-99, XI-99, XII-99, I-00, II-00, IV-00, V-00, VI-00 (350 fêmeas e 17 machos); PR 255 X-99, XI-99, XII-99, I-00, II-00, IV-00, V-00 (55 fêmeas e 4 machos); Pontes e Lacerda: IAN 873 IX-98, X-98, XI-98, XII-98, II-99, III-99, IV-99, V-99, VIII-99, IX-99, X-99, XI-99, XII-99, I-00, II-00, III-00, V-00, VI-00 (450 fêmeas e 10 machos); IAN 713 VIII-99, X-99, XI-99, XII-99, I-00, II-00, III-00, IV-00, V-00, VI-00 (85 fêmeas e 8 machos); FX 3864 VIII-99, IX-99, X-99, XI-99, XII-99, I-00, II-00, III-00, IV-00, V-00 (110 fêmeas e 6 machos); RRIM 600 VIII-99, IX-99, X-99, XI-99, XII-99, I-00, II-00, III-00, IV-00, V-00 (85 fêmeas e 6 machos).

Registros prévios no Brasil. Mato Grosso, Pará, São Paulo (BAKER 1945; FERES 2000)

Observações. Ácaro plano, conhecido no Estado do Pará como "ácaro-vermelho-da-seringueira" (FLECHTMANN 1979). Ocorre preponderantemente na face abaxial dos folíolos, sendo que em grandes populações pode ser encontrado também na face adaxial (FERES 2000).

\section{Tetranychidae Donnadieu, 1875}

Mononychellus sp.

Espécimes examinados. Itiquira: PB 260 XII-98, X-99 (3 fêmeas); PR 255 X-99, I-00 (3 fêmeas e 1 macho); Pontes e Lacerda: IAN 873 XI-98, VIII-99, XII-99 (3 fêmeas e 1 macho); IAN 713 VIII-99, X-99, XII-99 (4 fêmeas); FX 3864 VIII-99, XII-99 (6 fêmeas); RRIM 600 VIII-99, IX-99, X-99, XII-99 (9 fêmeas e 1 macho).

Observações. Esta é a primeira constatação de uma espécie deste gênero nos seringais do Brasil.

\section{Eutetranychus banksi (McGregor, 1914)}

Tetranychus banksi McGregor, 1914: 358.

Anychus banksi; McGregor, 1919: 644.

Eutetranycus banksi; McGregor, 1950: 268; Pritchard \& Baker, 1955: 115; Flechtmann \& Baker, 1970: 156; Flechtmann \& Baker, 1975: 112.

Espécimes examinados. Itiquira: PB 260 VIII-99 (1 fêmea); PR 255 IX-99 (1 macho).

Registros prévios no Brasil. Bahia, Mato Grosso, Minas Gerais e São Paulo (Silva 1972; Flechtmann \& Abreu 1973; Feres 2000). 
Observações. Nos Estados Unidos da América do Norte esta espécie é considerada séria praga dos citros. No Brasil, ocorre sobre grande número de plantas, sem que se tenha registrado danos de importância na cultura da seringueira (FERES 2000) ou em outras culturas.

\section{Oligonychus coffeae (Nietner, 1861)}

Acarus coffeae Nietner, 1861: 845.

Oligonychus coffeae; Pritchard \& Baker, 1955: 315; Baker \& Pritchard, 1960: 505; Meyer \& Rodrigues, 1965: 12; Rodrigues, 1968: 220; Gutierrez, 1968: 446; Meyer, 1974: 251; Meyer, 1987: 146.

Espécimes examinados. Itiquira: PB 260 II-00 (2 fêmeas); PR 255 II-00 (2 fêmeas e 1 macho); Pontes e Lacerda: IAN 873 IX-98, III-99, II-00 (3 fêmeas e 1 macho); IAN 713 XI-99 (1 fêmea); FX 3864 IX-99, I-00 (2 fêmeas e 1 macho).

Registros prévios no Brasil. Espírito Santo e Mato Grosso (FERES 2000; FLECHTMANN \& ARLEU 1984).

Observações. Espécie de coloração vermelha, registrada pela primeira vez no Brasil atacando seringueiras no Estado do Espírito Santo. Foram observados na face adaxial do folíolo, ao longo da nervura principal (FLECHTMANN \& ARLEU 1984).

\section{Oligonychus gossypii (Zacher, 1921)}

Paratetranychus gossypii Zacher, 1921: 183.

Oligonychus gossypii; Baker \& Pritchard, 1960: 508; Meyer, 1974: 263; Meyer, 1987: 152; Feres, 2000: 166.

Espécimes examinados. Itiquira: PB 260 XII-98, I-99, II-99, V-99, VIII-99, XII-99, I-00, II-00, IV-00, V-00, VII-00 (35 fêmeas e 6 machos); PR 255 VIII-99, XII-99, II-00, V-00, VII-00 (7 fêmeas); Pontes e Lacerda: IAN 873 IX-98, X-98, XI-98, XII-98, II-99, VIII-99, IX-99, X-99, XI-99, XII-99, II-00, IV-00, VI-00 (85 fêmeas e 4 machos); IAN 713 VIII-99, IX-99, X-99, XI-99, XII-99, I-00, II-00, III-00, IV-00, V-00 (30 fêmeas e 1 macho); FX 3864 VIII-99, IX-99, XI-99, XII-99, I-00, II-00, VI-00 (45 fêmeas); RRIM 600 VIII-99, IX-99, X-99, XI-99, XII-99, I-00, IV-00 (25 fêmeas e 2 machos).

Registros prévios no Brasil. Acre, Amazonas, Mato Grosso e São Paulo (FAZOLin \& PEREIRA 1989; FleChTMANn 1989; Feres 2000).

Observações. Registrada em vários países da África e nas Américas Central e do Sul, em várias espécies de plantas (FERES 2000). De cor avermelhada, localiza-se na face abaxial dos folíolos e produz pouca teia. Em altas populações, causa bronzeamento e desfolhamento prematuro (FLECHTMANN 1989; FAZOLIM \& PEREIRA 1989).

\section{Tetranychus mexicanus (McGregor, 1950)}

Septanychus mexicanus McGregor, 1950: 323.

Tetranychus mexicanus; Pritchard \& Baker, 1955: 411; Flechtmann \& Baker, 1970: 162; Flechtmann \& Baker, 1975: 120.

Espécimes examinados. Itiquira: PB 260 IX-98, XII-98, I-99, II-99, V-99, VI-99, VIII-99, X-99, XI-99, XII-99, II-00, IV-00, V-00, VII-00 (45 fêmeas e 3 machos); PR 255 VIII-99, X-99, XI-99, XII-99, I-00, II-00, VII-00 (13 fêmeas e 1 
macho); Pontes e Lacerda: IAN 873 IX-98, X-98, XII-98, III-99, V-99, VII-99, VIII-99, IX-99, XI-99 (28 fêmeas e 3 machos); IAN 713 VIII-99, IX-99, X-99, XI-99 (10 fêmeas e 2 machos); FX 3864 VIII-99, IX-99, XI-99 (14 fêmeas e 1 macho); RRIM 600 VIII-99, IX-99, X-99, I-00, VII-00 (16 fêmeas e 1 macho); IAN 713 VIII-99, IX-99, X-99, XI-99 (14 fêmeas e 2 machos).

Registros prévios no Brasil. Bahia, Mato Grosso, Minas Gerais, Pernambuco e São Paulo (FleChTMANN \& BAKER 1970; PASCHOAL 1971; FERES 2000).

Observações. Espécie de ampla distribuição geográfica, ocorrendo em grande número de plantas hospedeiras. Um aumento na população de ácaros pode ser observado logo após o enfolhamento, nos meses de julho e agosto, ocorrendo até o início das chuvas em Itiquira e Pontes e Lacerda. Verificou-se que o edeago dos espécimes coletados, embora semelhante na forma, é menor que o descrito para a espécie.

\section{Tydeidae Kramer, 1877}

\section{Lorryia formosa Cooreman, 1958}

Lorryia formosa Cooreman, 1958:6; Baker, 1968:995

Espécimes examinados. Itiquira: PB 260 IX-98, I-99, II-00 (17 fêmeas e 2 machos); Pontes e Lacerda: IAN 873 IV-99 (1 fêmea).

\section{Lorryia sp.}

Espécimes examinados. Itiquira: PB 260 II-99 (13 fêmeas e 2 machos); Pontes e Lacerda: IAN 873 IX-98, VII-00 (5 fêmeas).

\section{Parapronematus sp.}

Espécimes examinados. Itiquira: PB 260 XII-98, VIII-99 (2 fêmeas e 1 macho); PR 255 VIII-99 (1 fêmea); Pontes e Lacerda: IAN 873 X-98, XII-98, VIII-99, IX-99, X-99, XI-99 (14 fêmeas e 6 machos); IAN 713 VIII-99, X-99, VI-00 (3 fêmeas e 1 macho); FX 3864 VIII-99, IX-99, X-99, XI-99, XII-99, IV-00 (11 fêmea e 3 machos); RRIM 600 VIII-99, I-00 (2 fêmeas).

\section{Pronematus sp.}

Espécimes examinados. Itiquira: PB 260 XII-98 (1 fêmea); Pontes e Lacerda: IAN 873 X-98, VIII-99, IX-99 (3 fêmeas e 1 macho); IAN 713 VIII-99, IX-99 (3 fêmeas); FX 3864 VIII-99, IX-99, IV-00 (4 fêmeas e 1 macho); RRIM 600 VIII-99, IX-99, I-00, IV-00 (3 fêmeas e 2 machos).

\section{Stigmaeidae Oudemans, 1931}

\section{Agistemus floridanus Gonzalez, 1965}

Agistemus floridanus Gonzalez, 1965: 38.

Espécimes examinados. Itiquira: PB 260 XII-98, I-99, II-99, X-99, XII-99, I-00, II-00, V-00, VI-00, VII-00 (32 fêmeas e 4 machos); PR 255 X-99, XII-99,

I-00, II-00, IV-00, V-00, VI-00, VII-00 (24 fêmeas e 6 machos); Pontes e Lacerda: 
IAN 873 XII-98, III-99, IX-99 (3 fêmeas); IAN 713 IX-99, II-00, III-00, IV-00, V-00, VI-00 (7 fêmeas); FX 3864 IX-99 (1 fêmea); RRIM 600 IX-99, V-00, VII-00 (5 fêmeas).

Observações. É a primeira constatação desta espécie no Brasil.

\section{Zetzellia aff. yusti}

Espécimes examinados. Itiquira: PB 260 II-00 (1 fêmea); Pontes e Lacerda: IAN 713 I-00 (1 fêmea); RRIM 600 II-00 (2 fêmeas).

Observações. Difere de Zetzellia yusti por apresentar três setas no genu I, área do escudo da base da seta $a$ completamente ligado ao escudo dorso-central, solenídia I com $13 \mu \mathrm{m}$ e setas $e$ e le iguais ou mais longas que as demais. Trata-se, possivelmente, de uma espécie nova.

\section{Chave para diagnóstico dos taxa de ácaros deste estudo}

1. Tarso do palpo com apotele; tritosterno presente; estigmas localizados lateralmente entre as coxas III e IV e associados ao peritrema . . Parasitiformes . Subordem Gamasida . . Phytoseiidae . . . . . . . . . . . . 2

- Tarso do palpo sem apotele; tritosterno ausente; sem estigmas no segundo par de coxas . . Acariformes . . . . . . . . . . . . . . . . . . 16

2. Região podonotal do escudo dorsal com 4 pares de setas "laterais" $(j 3, z 2, z 4$ e s4) . Amblyseiinae . . . . . . . . . . . . . . . . 3

- Região podonotal do escudo dorsal com 6 pares de setas "laterais" ( $j 3, z 2, z 3, z 4$, $s 4$ e $s 6) \ldots$ Typhlodrominae . . . . . . . . . . . . . 13

3. Seta $J V I$ afastada da margem anterior do escudo ventrianal; peritrema normalmente curto, raramente estendendo-se além da base da seta $z 2$. Euseius . 4

- Seta $J V I$ inserida próxima à margem do escudo ventrianal; peritrema normalmente estendendo-se até a base da seta $j 1 \ldots \ldots \ldots \ldots \ldots \ldots \ldots \ldots \ldots \ldots \ldots \ldots \ldots \ldots$

4. Escudo dorsal com poucas estrias na região antero-lateral; seta $z 2$ menor que $z 4$, que por sua vez é menor que $s 4 \ldots \ldots \ldots \ldots \ldots \ldots$ Euseius concordis

- Escudo dorsal totalmente reticulado; comprimentos relativos das setas $z 2, z 4$ e $s 4$

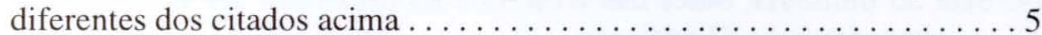

5. Macrosetas da perna IV com extremidade distal dilatada . . . . . Euseius alatus - Macrosetas da perna IV com extremidade distal afilada . . . . Euseius citrifolius

6. Macrosetas presentes apenas na perna IV . . Neoseiulus . . . . . . . . . . 7

- Macrosetas presentes também em outras pernas . . . . . . . . . . . 8

7. Maioria das setas do escudo dorsal curtas, não alcançando as bases das setas imediatamente posteriores; cálice da espermateca em forma de um curto sino; macroseta do tarso IV com $29 \mu \mathrm{m}$ de comprimento... Neoseiulus tunus

- Maioria das setas do escudo dorsal longas, geralmente ultrapassando a distância entre as bases das setas imediatamente posteriores; cálice da espermateca alongado; macroseta do tarso IV com $44 \mu \mathrm{m}$. . . . . . Neoseiulus anonymus 
8. Tarso I com seta proximal ereta; setas $Z 5$ consideravelmente maiores que a distância entre suas bases . . Amblyseius . . . . . . . . . . . . . . . 9

- Tarso I sem seta proximal ereta; setas Z5, relativamente curtas, usualmente mais curtas ou apenas um pouco mais longas que a distância entre suas bases . .10

9. Cálice da espermateca em forma de taça rasa ....... Amblyseius neochiapensis

- Cérvix da espermateca cilíndrico, com um alargamento no meio assemelhando-se a um anel, que divide o cérvix em uma metade mais longa, próxima à vesícula, e a outra metade mais estreita, próxima ao átrio . . . . . . . . . .

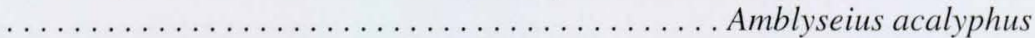

10. Margem posterior do escudo esternal trilobada pouco visível; escudo ventrianal com constrição lateral; setas $J V 1$ inseridas junto à margem anterior deste escudo . . Typhlodromalus . . . . . . . . . . . . . . . . 11

- Margem posterior do escudo esternal reta ou côncava, sempre bem visível; escudo ventrianal aproximadamente pentagonal; setas $J V I$ inseridas bem posterior à margem anterior deste escudo . . Typhlodromips . . . . . . . . . . 12

11. Escudo dorsal liso; setas do escudo dorsal afilando-se progressivamente da base ao ápice; macrosetas com pontas dilatadas . . . Typhlodromalus aff. horatii

- Escudo dorsal estriado antero-lateralmente; setas do escudo dorsal capitadas e lisas, exceto $S 5$ e $J 5$ que são setiformes; $Z 5$ serreada; macrosetas capitadas Typhlodromalus feresi

12. Escudo dorsal liso; setas do escudo dorsal lisas; setas Z4 e Z5 afilando-se progressivamente da base ao ápice; macrosetas afiladas .

Typhlodromips aff. sinensis

- Escudo dorsal reticulado; setas Z4 e Z5 grossas e serreadas; macrosetas com pontas

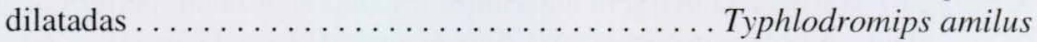

13. Seta $R I$ presente; setas $J V 3$ ausente; seta $S 4$ presente; quase todas as setas do escudo dorsal longas, serreadas e com dilatação no ápice . . . . . . . . . . ................... Typhlodromus (Anthoseius) transvaalensis

- Seta $R 1$ ausente; setas JV3 presente ou ausente; seta $S 4$ ausente; setas do escudo dorsal afilando-se progressivamente da base para o ápice . . . . . . . 14

14. Seta S5 diminuta; bases das setas $S 5$ e Z5 próximas; apenas as setas $Z 4$ e $Z 5$ serreadas; seta $Z I$ presente . . . . . . . . . . . . . . . . . . . . . . .

Galendromimus (Galendromimus) alveolaris

- Seta S5 longa; bases das setas S5 e Z5 distantes; todas as setas do escudo dorsal serrilhadas; seta $Z 1$ ausente . . . . . . . . . . . . . . 15

15. Peritrema curto, alcançando pouco além da base da seta $r 3$; setas do escudo dorsal longas; seta $R I$ ausente

. Galendromus (Galendromus) annectens De Leon

- Peritrema longo, alcançando a base da seta $j 1$; setas do escudo dorsal curtas; seta $R I$ presente $\ldots \ldots \ldots \ldots \ldots \ldots \ldots \ldots \ldots \ldots \ldots \ldots \ldots \ldots \ldots \ldots \ldots \ldots \ldots$ Galendromus $\mathrm{sp}$.

16. Empódio pode estar ausente; se presente, de formas variadas; sistema traqueal geralmente presente, com estigmas abrindo-se entre as bases das quelíceras 
ou na base do gnatossoma ou anteriormente no propodossoma; quelíceras estiletiformes, raramente queladas; abertura genital de diversas formas . . . . . .. Subordem Actinedida

- Empódio unciforme, situado em um distinto pré-tarso ou carúncula; unhas verdadeiras ausentes; sistema traqueal ausente; quelíceras quelado-denteadas; abertura genital em forma de V invertido . . . Subordem Acaridida . . 36

17. Gnatossoma em forma de cápsula ovóide; palpos diminutos; perna IV da fêmea com duas setas distais flageliformes . . Tarsonemidae . . . . . . . 18

- Gnatossoma sem a forma de cápsula; palpos distintos, se não distintos o corpo é vermiforme e o ácaro apresenta dois pares de pernas em todos os estágios; em alguns grupos pode apresentar palpo com seta dorsal da tíbia modificada em esporão, que desloca o tarso lateral ou ventralmente (complexo "unhadedão"); perna IV sem setas distais flageliformes

18. Tarso IV do macho terminando em um robusto esporão; escudo prodorsal da fêmea cobre os estigmas dorsolaterais; setas dorsais de diferentes tamanhos Tarsonemus

- Tarso IV do macho terminando numa estrutura arredondada; escudo prodorsal da fêmea não cobre os estigmas dorsolaterais; setas dorsais curtas . . . . . . . . . Polyphagotarsonemus latus

19. Corpo vermiforme; com dois pares de pernas . . Eriophyidae . . . . . . . 20

- Corpo ovalado, com mais de 2 pares de pernas . . . . . . . . . . . . . 22

20. Escudo dorsal sem setas, com margem anterior e laterais reticuladas; empódio dividido em 4 segmentos . . . . . . . . . . Calacarus heveae Feres

- Escudo dorsal com setas; margens do escudo não reticuladas; empódio dividido

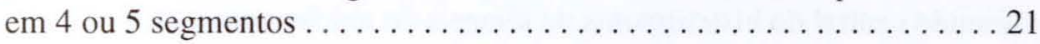

21. Tubérculos dorsais localizados junto à margem posterior do escudo dorsal; empódio dividido em 5 segmentos; anéis do opistossoma uniformemente

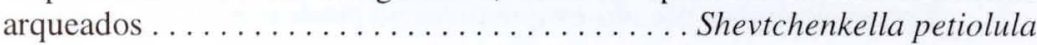

- Tubérculos dorsais localizados anteriormente à margem posterior do escudo dorsal; empódio com 4 segmentos; anéis do opistossoma com largo sulco dorso-mediano . .................. Phyllocoptruta seringueirae

22. Palpo com seta dorso-distal da tíbia modificada em esporão, que desloca o tarso lateral ou ventralmente (complexo "unha-dedão") . . . . . . . . . . 23

- Palpo sem o complexo "unha dedão" . . . . . . . . . . . . . . . . . 30

23. Quelíceras com estiletes longos e recurvados, implantados em um estilóforo; tarsos I e II com setas dúplices ou associadas; região ao redor da abertura genital pregueada . . Tetranychidae . . . . . . . . . . . 24

- Quelíceras curtas e afiladas, não implantadas em estilóforo; tarsos sem setas dúplices ou associadas; região ao redor da abertura genital da fêmea não

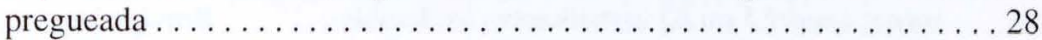


24. Empódio ausente ou dividido distalmente; dois pares de setas paranais . . . 25

- Empódio unciforme; um par de setas paranais . . . . . . . . . . . . . 26

25. Tarso I sem setas dúplices, mas com setas associadas; empódio ausente ...... Eutetranychus banksi

- Tarso I com setas dúplices distais e próximas; empódio fendido distalmente .... Mononychellus sp.

26. Tarso I com setas dúplices bem separadas no segmento . .

Tetranychus mexicanus

- Tarso I com setas dúplices distais e próximas; unha do empódio com cerdas próximo-ventrais . . Oligonychus Berlese . . . . . . . . . . . 27

27. Tibia I da fêmea com sete setas táteis; edeágo dirigido ventralmente terminando

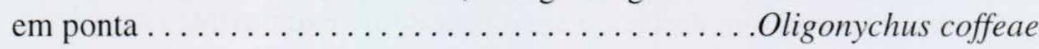

- Tíbia I da fêmea com nove setas táteis; edeago dirigido dorsalmente, com cabeça sigmóide .......................... Oligonychus gossypii

28. Bases das quelíceras fundidas com o restante do gnatossoma; tarso do palpo com seta modificada robusta e em forma de foice, tarso do palpo com duas setas pectinadas; segmentos do palpo mais robustos que os das pernas ... Cheyletidae .............................. Hemicheyletia

- Bases das quelíceras totalmente separadas ou parcialmente fundidas, porém sempre independentes do restante do gnatossoma; tarso do palpo sem setas modificadas em forma de foice e tarso do palpo sem setas pectinadas; segmentos do palpo de diâmetro semelhante ao das pernas ... Stigmaeidae

29. Escudo central do histerossoma com 5 pares de setas na fêmea e 6 no macho. . Agistemus floridanus

- Escudo central do histerossoma da fêmea e do macho com 4 pares de setas ..... Zetzellia aff yusti

30. Palpo de comprimento aproximadamente igual ao das quelíceras; dorsalmente, 2 pares de órgãos pseudo-estigmáticos no prodossoma ...... Cunaxidae . . Pseudobonzia sp.

- Bases das quelíceras fundidas podendo formar um estilóforo; somente um par de setas sensoriais no dorso do propodossoma ou sem essas setas ........ 31

31. Corpo achatado dorso-ventralmente; dígitos móveis das quelíceras modificados em estiletes longos e recurvados; setas sensoriais propodossomais ausentes ..... Tenuipalpidae ............................. 32

- Corpo não achatado; dígitos móveis das quelíceras modificados em estiletes curtos e retos; com um par de setas sensoriais no dorso do propodossoma ...... Tydeidae ................................... 33

32. Palpo com 3 segmentos; podossoma tipicamente mais largo que o opistossoma; setas $L 5$ ou $h 2$ flageliforme ................ Tenuipalpus heveae

- Palpo com 4 segmentos; podossoma não distintamente mais largo que o opistossoma; setas $L 5$ ou $h 2$ semelhantes às demais ....... Brevipalpus phoenicis 
33. Estrias dorsais do tegumento formando padrão reticulado na totalidade ou parte do idiossoma . . . . . . . . . . . . . . . . . . . . . . 34

- Estrias dorsais do tegumento não formam padrão reticulado . . . . . . . . . 35

34. Setas dorsais lanceoladas e lisas, com metade apical tipicamente expandida e recurvada ........................... Lorryia formosa - Setas dorsais fortemente serrilhadas, sem expansão na metade apical da seta .... Lorryia sp.

35. Setas anais ausentes; fêmures III e IV apresentando, cada um, uma proeminente seta bifurcada ......................... Parapronematus sp. - Setas anais presentes; fêmures III e IV sem setas proeminentes bifurcadas . . . . . Pronematus sp.

36. Empódio unciforme ligado à extremidade do tarso por um delicado tendão ... Wintershmidtiidae ....................... Oulenzia $\mathrm{sp.}$

36’Empódio unciforme ligado à extremidade do tarso por um par de estruturas em forma de bastão . . Acaridae . . . . . . . . . . . . . . . . . . . 37

37. Setas ve pequenas e finas, implantadas próximo da altura mediana do bordo lateral do escudo dorsopropodossomal; setas sce são mais longas que sci . . Neotropacarus sp.

- Setas ve longas e implantadas quase à mesma altura que as setas vi; setas sce são mais curtas do que $s c i \ldots \ldots \ldots \ldots \ldots \ldots \ldots \ldots$ Tyrophagus $\mathrm{sp}$.

\section{DISCUSSÃO}

Este estudo constatou uma grande diversidade de ácaros na cultura da seringueira nos dois locais pesquisados no Estado do Mato Grosso, sendo que Pontes e Lacerda apresentou 32 espécies e Itiquira, 28 espécies.

Os ácaros mais freqüentemente encontrados pertencem à subordem Actinedida, sendo mais comuns aqueles das famílias Eriophyidae, Tenuipalpidae e Tetranychidae.

Dentre as espécies de eriofídeos coletadas, $C$. heveae e $P$. seringueirae foram as mais frequientes. Entretanto, $C$. heveae foi mais comum em Itiquira e $P$. seringueirae, em Pontes e Lacerda. As duas espécies foram coletadas durante todo o ano nos diferentes clones avaliados. Possivelmente, a diferença de freqüência se deve aos clones cultivados e às condições climáticas distintas, apresentadas pelos dois locais. Devido à baixa freqüência, $S$. petiolula parece ser de pouca importância econômica para a cultura da seringueira.

Dentre os Actinedida, a maior diversidade foi apresentada pelas famílias Tetranychidae e Eriophyidae, com cinco espécies cada, aparecendo em seguida os tideídeos, com quatro espécies. Entre os tetraniquídeos, T. mexicanus e $O$. gossypii foram as espécies mais freqüentemente encontradas nas duas fazendas. Pela primeira vez é relatada a presença de uma espécie do gênero Mononychellus nesta cultura.

Tenuipalpus heveae e $B$. phoenicis foram as únicas espécies da família Tenuipalpidae encontradas. A frequiência $T$. heveae foi alta durante todo $o$ ano nas duas fazendas, enquanto que $B$. phoenicis apresentou baixa frequiência nos dois 
municípios. T. heveae éconsiderado o tenuipalpídeo mais importante na cultura da seringueira.

Dentre os predadores da subordem Actinedida foram encontrados ácaros das famílias Cunaxidae, Tydeidae (Subfamília Pronematinae) e Stigmaeidae. Os estigmeídeos A. floridanus e Zetzellia aff. yusti foram encontrados nos dois locais amostrados, sendo A. floridanus a espécie mais freqüente. A maior freqüência de A. floridanus foi em Itiquira. Os ácaros da família Cunaxidae foram comuns em Pontes e Lacerda, sendo que a única espécie encontrada pertenceu ao gênero Pseudobonzia.

A família Tydeidae apresentou grande diversidade. Entretanto, poucos indivíduos foram encontrados. A maior freqüência foi constatada em Pontes e Lacerda, pelos ácaros dos gêneros Pronematus e Parapronematus. Estes são referidos por LAING \& KNOP (1982) e PERRIN \& MCMURTRY (1996) como ácaros predadores de eriofídeos.

Na subordem Gamasida foram coletados apenas ácaros da família Phytoseiidae. Até o momento, apenas oito espécies de fitoseídeos haviam sido relatadas nos seringais do Estado do Mato Grosso (FERES 2000). O presente trabalho relata mais 10 espécies. Entretanto, o número de espécies encontradas em Pontes e Lacerda (14) foi o dobro daquelas encontradas em Itiquira (7). Possivelmente, as condições climáticas de Pontes e Lacerda, ambiente próximo da Amazônia, local de onde é originária a seringueira, tenham possibilitado a presença de uma maior diversidade de fitoseídeos. É possível também, que essa diferença possa estar ligada aos diferentes clones estudados em Pontes e Lacerda.

Os representantes da subordem Acaridida das famílias Acaridae e Wintershmidtiidae estiveram presentes apenas em Itiquira e com baixa freqüência. Suas ocorrências foram restritas aos meses de janeiro a julho, sendo sua maior frequiência observada entre abril e junho, época em que as folhas estão velhas, com concentração de fungos, liquens e matéria orgânica, o que propicia o desenvolvimento de populações destas famílias (FLECHTMANN 1986).

AGRADECIMENTOS. Aos Profs Drs Reinaldo J.F. Feres e Carlos H.W. Flechtmann pelo auxílio na identificação das espécies encontradas. Às empresas Plantações Edouard Michellin Ltda. e Triângulo Agro-Industrial Ltda pelo financiamento do projeto. Ao Engenheiro Agrônomo Caio Franchechi, ao Técnico Agrícola Nilson de Souza e aos Engenheiros Agrônomos Cássio Scomparin e Etiéne Grallien pelo envio do material para a realização destes estudos. Ao CNPq pela concessão de bolsa para a realização do doutorado do primeiro autor.

\section{REFERÊNCIAS BIBLIOGRÁFICAS}

Amrine, J.R. \& D.C.M. MAnson. 1996. Preparation, Mounting and Descriptive Study of Eriophyoid Mites, p. 383-396. In: E.E. Lindquist; M.W. Sabelis \& J. Bruin (Eds). Eriophyoid Mites. Amsterdam, Elsevier, 643p.

BANKs, N. 1904. Class III, Arachnida, Order I, Acarina, four new species of injurious mites. Jour. N.Y. Entomol. Soc. 12: 53-56.

Baker, E.W. 1945. Mites of the genus Tenuipalpus (Acarina: Trichadenidae). Proc. Entomol. Soc.

Wash. 47 (2): 33-44. 
1968. The genus Lorryia. Ann. Entomol. Soc. Amer. 61 (4): 986-1008.

BAKER, E.W. \& A.E. PritchaRd. 1953. A review of the false spider mite genus Tenuipalpus Donnadieu (Acarina: Phytoptipalpidae). Ann. Entomol. Soc. Amer. 46 (3): 317-336.

1960. The tetranychoid mites of Africa. Hilgardia, Berkeley, 29 (11): 455-574.

Baker, E.W. \& D.M. Tuttle. 1987. The false spider mites of Mexico (Tenuipalpidae: Acari). Tech. Bull. U.S. Dep. Agric. 1706: 1-237.

BAKER, E.W.; D.M. TutTLE, \& M.J. AbBatiello. 1975. The false spider mites of northwestern and north central Mexico (Acarina: Tenuipalpidae). Smith. Contrib. Zool. 194: 1-23.

Berlese, A. 1913. Acari nuovi. Manipoli VII-VIII. Redia, Firenze, 9: 77-111.

1916. Centuria prima di Acari nuovi. Redia, Firenze, 12: 19-66.

CHANT, D.A. 1955. Notes on mites of the genus Typhlodromus Scheuten, 1857 (Acarina: Laelaptidae), with descriptions of the males of some species and the female of a new species. Can. Entomol. 87 (11): 496-503.

- 1959. Phytoseiid mites (Acarina: Phytoseiidae). Part I. Bionomics of seven species in southeastern England. Part II. A taxonomic review of the family Phytoseiidae, with descriptions of thirthy-eight new species. Can. Entomol. 91. (Suppl. 12): 1-166.

Chant, D.A. \& E.W. Baker. 1965. The Phytoseiidae (Acarina) of Central America. Mem. Entomol. Soc. Can. 41: 1-56.

Chant, D.A. \& J.A. McMurtry. 1994. A review of the subfamilies Phytoseiinae and Typhlodrominae (Acari: Phytoseiidae). Internat. Jour. Acarol. 20 (4): 222-311.

Chant, D.A. \& E. YoshidA-Shaul. 1983. A world review of the simplex species groups in the genus Typhlodromus Scheuten: Part II. The conspicuus and cornus groups (Acarina:Phytoseiidae). Can. Jour. Zool. 61: 1041-1057.

-1984. A world review of the occidentalis species group in the genus Typhlodromus Scheuten (Acarina: Phytoseiidae). Can. Jour. Zool. 62: 1860-1871.

1986a. A world review of the eclesiasticus group in the genus Typhlodromus Scheuten (Acarina: Phytoseiidae) Can. Jour. Zool. 64: 447-466.

1986b. A new subfamily, Cydnodromellinae, in the family Phytoseiidae (Acari: Gamasina) Can. Jour. Zool. 64: 2811-2823.

Chiavegato, L.G. 1980. Ácaros da cultura dos citros, p. 469-501. In: O. Rodriguez \& F.C.P. ViéGas (Eds). Citricultura brasileira. Campinas, Fundação Cargill, 739p.

Cooreman, J. 1958. Notes et observations sur les Acariens. VII-Photia graeca n.sp. (Acaridia, Canestriniidae) et Lorryia formosa n.sp. (Stomatostigmata, Tydeidae). Bull. Inst. Roy. Sci. Nat. Entomol. 41: 73-76.

DeLeON, D. 1957. Three new Typhlodromus from southern Florida (Acarina: Phytoseiidae). Florida Entomol. 40: 141-144.

1958. Four new Typhlodromus from southern Florida (Acarina: Phytoseiidae). Florida Entomol. 41: 73-76.

1961. The genus Brevipalpus in Mexico. Part II (Acarina: Temuipalpidae). Florida Entomol. 44 (1): 41-52.

-1962a. The cervices of some phytoseiid type specimens (Acarina: Phytoseiidae). Acarologia 4: $174-176$

. 1962b. Twenty-three new Phytoseiidae, mostly from southeastern United States (Acarina: Phitoseiidae). Florida Entomol. 45 (1): 11-27.

- 1966. Phytoseiidae of British Guyana with keys to species (Acarina: Mesostigmata). In: Studies on the fauna of Suriname and other Guyanas 8: 81-102.

- 1967. Some mites of the Caribbean Area. Part I. Acarina on plants in Trinidad, West Indies. Lawrence, Kansas, Allen Press Inc., 66p.

Denmark, H.A. \& M.H. Muma. 1970. Some Phytoseiidae mites of Paraguay Phytoseiidae: Acarina).

Florida Entomol. 53 (4): 219-227.

1973. Phytoseiid mites of Brazil (Acarina: Phytoseiidae). Rev. Brasil. Biol. 33: 235-276.

Revta bras. Zool. 19 (3): $867-888,2002$ 
1989. A revision of the genus Amblyseius Berlese, 1914 (Acari: Phytoseiidae). Occasional

Papers Florida Sta. Coll. Arthropods 4: 1-149.

Donnadieu, A.L. 1875. Recherches pour servir à l'histoire des Tétranyches. Ann. Soc. Linn. Lyon 22: 29-136.

EMBRATER. 1983. Cultura da seringueira. Brasília, EMBRATER, 135p.

EwING, H.E. 1939. A revision of the mites of the subafamily Tarsoneminae of North America, the West Indies and the Hawaiian Islands. Tech. Bull. U.S. Dept. Agric. 653: 1-63.

Farias, A.R.; C.H.W. Flechtmann; G.J. Moraes \& J.A. MCMurTRY. 1981. Predadores do ácaro verde da mandioca, no nordeste do Brasil. Pesq. Agropec. Brasileira, Brasília, 16 (3): 313-317.

Fazolin, M. \& L.V. Pereira. 1989. Ocorrência de Oligonychus gossypii (Zacher, 1920) (Acari: Tetranichidae) em seringueiras cultivadas. An. Soc. Entomol. Brasil 18 (1): 199-202.

FERES, R.J.F. 1992. A new species of Calacarus Keifer (Acari, Eriophyidae, Phyllocoptinae) from Hevea brasiliensis Muell. Arg. (Euphorbiaceae) from Brazil. Internat. Jour. Acarol. 18 (1): 61-65.

1998. Two new Phyllocoptinae mites (Acari, Eriophyidae,) from Hevea brasiliensis Muell. Arg. (Euphorbiaceae) from Brazil. Internat. Jour. Acarol. 24 (1): 69-74.

. 2000. Levantamento e observações naturalísticas da acarofauna (Acari, Arachnida) de seringueiras cultivadas (Hevea spp., Euphorbiaceae) no Brasil. Revta bras. Zool. 17 (1): 157-173.

Feres, R.J.F. \& G.J. DE Moraes. 1998. Phytoseidae mites (Acari: Phytoseiidae) from woody areas in the State of São Paulo, Brazil. Sist. Appl. Acarol. 3: 125-132.

FERLA, N.J. \&. G.J. DE MoraEs. 1998. Ácaros predadores em pomares de maçã no Rio Grande do Sul. An. Soc. Entomol. Brasil 27 (4): 649-654.

Flechtmann, C.H.W. 1975. Elementos de Acarologia. São Paulo, Livraria Nobel, 344p.

1976. A report on the Tetranychidae (Acari) of Brasil: an emmendation. Revta bras. Ent.

20 (2): 115-116.

1979. Tuckerella ornata (Tucker), um ácaro novo para o Brasil e outros Tetranychoidea

(Acari) do Estado do Pará. An. Esc. Sup. Agric. Luiz de Queiroz, Piracicaba, 36: 615-620.

1986. Ácaros em produtos armazenados. Piracicaba, Fundação de Estudos Agrários Luiz de Queiroz, 97p.

. 1989. Seringueira (Hevea sp.) um novo hospedeiro para Oligonychus gossypii (Zacher, 1920)

(Acari: Tetranychidae). Bol. Mus. Paraense Emílio Goeldi, sér. zool., 5 (1): 127-128.

Flechtmann, C.H.W. \& J.M. Abreu. 1973. Ácaros fitófagos do Estado da Bahia, Brasil. Ci. Cult. 25 (3): $244-251$

Flechtmann, C.H.W.\& R.J. ARLEU. 1984. Olygonychus coffeae (Nietner, 1961), um ácaro tetraniquídeo da seringueira (Hevea brasiliensis) novo para o Brasil e observações sobre outros ácaros desta planta. Ecossistema 9: 123-125.

FlechtMAnN, C.H.W. \& E.W. BAKER. 1970. A preliminary report on the Tetranychidae (Acarina) of Brazil. Ann. Entomol. Soc. Amer. 63 (1): 156-163.

1975. A report on the Tetranychidae (Acari) of Brazil. Revta bras. Ent. 19 (3): 111-122.

GravenA, S. 1994. Manejo integrado de pragas em citros: uma visão atual, p. 41-56. In: L.C. Donadio

\& S. Gravena (Eds). Manejo integrado de pragas dos citros. Campinas, Fundação Cargill, 310p.

GeIJSKES, D.C. 1939. Beitraege zur Kenntnis der europaeischen Spinnmilben (Acari: Tetranychidae), mit besonderer Beruecksichtigung der niederlaendischen Arten. Meded. Landbouwhoogesc. Wageningen Nederland 42 (4): 1-68.

Gonzales, R.H. 1965. A taxonomic study of the genera Mediolata, Zetzellia and Agistemus. Univ. California Publ. Entomol. 41: 1-64.

_ 1975. Revision of the Brevipalpus phoenicis "complex", with descriptions of new species from Chile and Thailand (Acarina: Tenuipalpidae). Acarologia 17 (1): 82-91.

Gutierrez, J. 1968. Tetranychidae nouveaux de Madagascar (Quatriéme note). Acarologia 10 (1): 13-28.

JEPSON, L.R.; KEIFER, H.H \& E.W. BAKER. 1975. Mites injurious to economic plants. Berkeley, Univ. California Press, XXIV+641p. 
Kramer, P. 1877. Grundzuge zur systematik der Milben. Arch. Naturg. 43: 215-247.

Kreiter, S. \& G.J. DE Moraes. 1997. Phytoseiid mites (Acari: Phytoseiidae) from Guadalupe and Martinique. Florida Entomol. 80 (3): 376-382.

LAING, J.E. \& N.F. KNOP. 1982. Potential use of predaceous mites other than Phytoseiidae for biological control of Orchards pests, p. 28-35. In: M.A. HoY; G.L. Cunningham \& L. KNUTSON. Biological control of pests by mites. Berkeley, Univ, California Press, 185p.

LEACH, W.E. 1815. A tabular view of the external characters of four classes of animals, etc. Trans. Linn. Soc. London 11: 399.

LINDQUIST, E.E. 1986. The world genera of Tarsonemidae (Acari: Heterostigmata): A morphological, phylogenetic, and systematic revision, with a reclassification of family-group taxa in the Heterostigmata. Mem. Ent. Soc. Can. 136: 1-517.

Lofego, A.C.; G.J. De Moraes \& J.A. McMurtry. 2000. Three new species of Phytoseiid mites (Acari: Phytoseiidae) from Brazil. An. Soc. Entomol. Brasil 29 (3): 461-467.

McGregor, E.A. 1914. Four new tetranychids. Ann. Entomol. Soc. Amer. 7: 354-364.

1919. The red spiders of America and a few European species likely to be introduced. Proc. U.S. Nat. Mus. 56: 641-579.

McGregor, E.A. 1950. Mites of the family Tetranychidae. Amer. Midl. Nat. 44 (2): 257-420.

MCMurtry, J.A. 1983. Phytoseiid mites from Guatemala, with descriptions of two new species and redefintions of the genera Euseius, Typhloseiopsis, and the Typhlodromus occidentalis species group (Acari: Mesostigmata). Internat. Jour. Entomol. 25: 249-272.

MEYER, M.K.P.S. 1974. Arevision of the Tetranychidae of Africa (Acari) with a key to the genera of the world. Entomol. Mem. Dep. Agric. Tech. Serv. Repub. S. Afr. 36: 1-291.

1979. The Tenuipalpidae (Acari) of Africa with keys to the world fauna. Entomol. Mem. Dept. Agric. Tech. Serv. Repub. S. Afr. 50: 1-135.

- 1987. African Tetranychidae (Acari: Prostigmata), with reference to the world genera. Entomol. Mem. Dept. Agric. Tech. Serv. Repub. S. Afr. 69: 1-175.

MeYer, M.K.P.S. \& M. DA C. RodRigUez. 1965. Acari associated with cotton in Southern Africa (with reference to the other plants). Garcia de Orta Lisboa 13 (2): 195-226.

MoraEs, G.J. DE. 1991. Controle biológico de ácaros fitófagos. Informe Agropec., Belo Horizonte, 15: $55-62$.

Moraes, G.J. DE; J. A. Alencar; F. Wenzel Neto, F. 1988. Explorations for natural enemies of the cassava green mite in Brazil. In: Proc. VIII Symp. Internat. Soc. Tropical Root Crops, Bankok, p. $351-353$.

Moraes, de G. J.; H.A. Denmark \& J. M. Guerrero. 1982. Phytoseiid mites of Colombia (Acarina: Phytoseiidae). Internat. J. Acarol. 8 (1): 15-22.

Moraes, G.J. De, S. Kreiter; A.C. Lofego. 2000. Plant mites (Acari) of the French Antilles. 3. Phytoseiidae (Gamasida). Acarologia 40 (3): 237-264.

Moraes, G.J. DE \& J.A. McMurTRy. 1983. Phytoseiid mites (Acarina) of northeastern Brazil, with descriptions of four new species. Internat. Jour. Acarol. 9: 131-148.

Moraes, G.J. DE; J.A. McMurtry \& H.A. Denmark. 1986. A catalog of the mite family Phytoseiidae: references to taxonomy, synonymy, distribution and habitat. Brasília, EMBRAPA-DDT, 353p.

Moraes, DE G.J. \& N.C. MESA. 1988. Mites of the family Phytoseiidae (Acari) in Colombia, with descriptions of three new species. Internat. Jour. Acarol. 14: 71-88.

Moraes, De G.J.; N.C. Mesa \& A. Braun. 1991. Mites of the family Phytoseiidae (Acari) in Colombia, with descriptions of three new species. Internat. Jour. Acarol. 14: 117-139.

Moraes, G.J. DE \& J.V. DE Oliveira. 1982. Phytoseiidae mites of coastal Pernambuco in northeasthern Brazil. Acarologia 23 (4): 315-318.

Muma, M.H. 1961. Subfamilies, genera, and species of Phytoseiidae (Acarina: Mesostigmata). Bull. Florida State Mus. Biol. Sci. 5: 267-302.

Muma, M.H.; H.A. Denmark \& D. DeLeon. 1970. Phytoseiidae of the Florida. Arthropods of Florida \& neighboring land areas. 6. Gainesville, Florida Dep. Agric., 150p. 
NALEPA, A. 1898.Zur Kenntniss der Gattung Trimerus Nal. Zoologische Jahrbuecher 11 (5): 402-411. NesBITT, H.H.J. 1951. A taxonomic study of the Phytoseiinae (Family Laelapidae) Predaceous upon Tetranychidae of economic importance. Zool. Verhandel. 12: 1-64.

NIETNER, J. 1861. Observation on the enemies of the coffee tree in Ceylon. 31p.

Oudemans, A.C. 1923. Studie over de sedert 1877 ontworpen systemen der Acari; Nieuve classificatie; Phylogenetische Beschouwingen. Tijdschrift voor Entomologie 66: 49-85.

- 1931. Eene nomenclatorische, tevens type-kwestie. Entomol. Ber., Amsterdam, 8: 189-204.

Paschoal, A.D. 1971. Nova relação de ácaros de plantas do Brasil. Revta. Per. Entomol. 14 (1):174-176

Perrin, T.M. \& J.A. MCMurtry. 1996. Other predatory arthropods, p.471-479. In: E.E. LindQuist; M.W. SABelis; J. BRuin (Eds). World crop pests. Eriophyoid mites - their biology, natural enemies and control. Amsterdam, Elsevier Society, 643p.

PRITChaRd, A.E. \& E.W. BAKER. 1955. A revision of the spider mite family Tetranychidae. San Francisco, Pacific Coast Entomol. Soc., Memoirs series, Vol. 2, 472p.

- 1958. The false spider mites (Acarina: Tenuipalpidae). Univ. California Publ. Entomol. 14 (3): $175-274$.

Reis, P.R.; L.G. Chiavegato; E.B. Alves \& E.O. Sousa. 2000. Ácaros da família Phytoseiidae associados aos citros no município de Lavras. Sul de Minas Gerais. An. Soc. Entomol. Brasil 29 (1): $95-104$.

Rodrigues, M. DA C. 1968. Acarina de Moçambique - Catálogo das espécies relacionadas com a agricultura. Agron. Moçamb. 2 (4): 215-256.

SAYED, M.T. 1946. Description of Tenuiapalpus granati nov. spec. and Brevipalpus pyri nov. spec. Bull. Soc. Fouad Entomol., Cairo, 30: 99-104.

SCHICHA, E. \& M. ELSHAFIE. 1980. Four new species of phytoseiid mites from Australia, and three species from America redescribed (Acari: Phytoseiidae). Jour. Australian Entomol. Soc. 19: 27-36.

Silva, P. 1972. Pragas da seringueira no Brasil, problemas e perspectivas. In: An. I Semin. Nac. Seringueira, Cuiabá, p.143-152.

Smiley, R.L. 1967. Some Tarsonemidae from the Republic of the Congo (Acarina). Proc. Entomol. Soc. Wash. 66: 145-150.

1992. Cunaxidae (Acari) of the World with a new classification. Michigan, Indira Publishing House, 356p.

ZaCHER, F. 1921. Neue und wenig bekannte Spinnmilben. Ztschr. Ang. Entomol., Amsterdam, 7: 181-187.

Recebido em 09.XI.2001; aceito em 04.IX.2002. 